\title{
LAF 1.0: IMPLANTAÇÃO DE UM SISTEMA INFORMATIZADO PARA LABORATÓRIOS DE ANTROPOLOGIA FORENSE
}

\author{
LAF 1.0: ESTABLISHMENT OF A COMPUTERIZED SYSTEM FOR \\ LABORATORIES OF FORENSIC ANTHROPOLOGY
}

\author{
Luís G. Crocco ${ }^{1}$, Guilherme de O. Brondi ${ }^{1}$, Andjara T.C. Soares ${ }^{2}$, Joaquim C. Felipe ${ }^{3}$, \\ Martin P. Evison ${ }^{4}$, Marco A. Guimarães ${ }^{5}$
}

\begin{abstract}
${ }^{1}$ Aluno de graduação do curso de Informática Biomédica, Faculdade de Medicina de Ribeirão Preto (FMRP-USP) e Faculdade de Filosofia, Ciências e Letras de Ribeirão Preto (FFCLRP-USP). ${ }^{2}$ Pós-graduanda do Centro de Medicina Legal, Departamento de Patologia, FMRP-USP. ${ }^{3}$ Docente do Departamento de Física e Matemática, FFCLRP-USP. ${ }^{4}$ Docente, Diretor do Forensic Science Program - University of Toronto at Mississauga, Canadá. ${ }^{5}$ Docente do Centro de Medicina Legal, Departamento de Patologia, FMRP-USP. CORRESPONDÊNCIA: Prof. Dr. Marco Aurelio Guimarães. Centro de Medicina Legal (CEMEL), Departamento de Patologia, Faculdade de Medicina de Ribeirão Preto - USP. Rua Tenente Catão Roxo no 2418, Ribeirão Preto, São Paulo, CEP:14051-140, Brasil.

Tel.: +55-16-36023358; fax: +55-16-36334476 / E-mail: mag@fmrp.usp.br
\end{abstract}

Crocco LG, Brondi GO, Soares ATC, Felipe JC, Evison MP, Guimarães MA. LAF 1.0: Implantação de um sistema informatizado para laboratórios de Antropologia Forense. Medicina (Ribeirão Preto) 2008; 41 (1): 12-16.

RESUMO: Objetivo: Desenvolvimento de um software para cadastro e recuperação de informações em Antropologia Forense baseado no protocolo desenvolvido durante o projeto "UK Brazil Scientific Cooperation - Forensic Anthropology and Identification of Human Remains". Metodologia: Por se tratar de um aplicativo acessado via Browser (software que permite o acesso à Internet, como o Microsoft Internet Explorer ${ }^{\circledR}$ ) foi necessária a escolha de uma linguagem de programação que se enquadrasse nesse requisito juntamente com uma aplicação servidora. $A$ linguagem escolhida foi $\mathrm{PHP} \circledast$ e a aplicação servidora foi o Apache ${ }^{\circledR}$. Para o armazenamento dos dados foi escolhido o Sistema Gerenciador de Banco de Dados MySQL®. Resultados: Testes realizados com dados reais comprovaram a eficiência do LAF 1.0 em se tratando de segurança, confiabilidade no armazenamento e recuperação dos dados além de satisfação do usuário, com o visual limpo e agradável das interfaces. Esta versão foi registrada no Instituto Nacional de Propriedade Intelectual (INPI) e encontra-se disponível gratuitamente para uso em todos os serviços médico-legais interessados em implantar um laboratório de Antropologia Forense. Conclusões: O software agilizou a geração de laudos de exames antropológicos para o Laboratório de Antropologia Forense do Centro de Medicina Legal (CEMEL) e permitiu a criação de uma ferramenta informatizada gratuita para uso em outros serviços semelhantes, o que permitirá maior facilidade na capacitação de pessoal para o trabalho em Antropologia Forense. $O$ software encontra-se atualmente em sua versão de atualização, denominada LAF 2.0.

Descritores: Medicina Legal. Antropologia Forense. Ossadas. Software. 


\section{1- INTRODUÇÃO}

A violência é um sério problema de saúde pública da atualidade, principalmente em países em desenvolvimento ou subdesenvolvidos. No Brasil, os dados de homicídios vêm crescendo de maneira alarmante. Em 1980, 117 pessoas em cada 100 mil morriam por homicídios. Em 2003, esse número atingiu 288 para cada 100 mil habitantes ${ }^{1}$.

Na região de Ribeirão Preto, um estudo retrospectivo realizado de 1993 a 1997 pelo Centro de Medicina Legal (CEMEL) da Faculdade de Medicina de Ribeirão Preto da Universidade de São Paulo (FMRPUSP) mostrou que a incidência de homicídios é maior entre os homens, ocorre principalmente através de armas de fogo e apresentou um aumento de 86,6\% neste intervalo de cinco anos. Apesar do número de homicídios ser menor entre as mulheres, foi observado um aumento de 51,3\% em apenas um ano, a maioria ocorrendo também por armas de fogo $(71,4 \%)^{2}$.

Este crescente número de homicídios pode estar relacionado com o crescimento econômico da cidade de Ribeirão Preto e com o crescimento populacional das cidades ao seu redor. A principal atividade econômica da região é a produção de açúcar e álcool e, em virtude disso, as plantações de cana-de-açúcar são abundantes. Entretanto, esses locais são esconderijos muito utilizados para corpos, que serão encontrados apenas no período de colheita da cana-de-açúcar.

Nesta época do calendário agrícola muitos corpos são encontrados ${ }^{3}$, em sua grande maioria em avançado estado de decomposição. Por muito tempo, devido à falta de recursos adequados para realização de exame antropológico, estas ossadas eram enterradas sem identificação.

O exame antropológico consiste na organização, análise e registro de dados referentes a ossadas de indivíduos não-identificados de forma a obter informações sobre as mesmas, tais como sexo, ancestralidade, idade, estatura, destreza manual, características odontológicas, sinais anatômicos particulares, causa da morte, entre outras, com a finalidade de facilitar o processo de identificação do corpo.

Diante da impossibilidade que havia de identificar essas ossadas, foi estabelecido um acordo entre a FMRP-USP e a University of Sheffield (UK) que viabilizou o desenvolvimento do projeto "UK - Brazil Scientific Cooperation - Forensic Anthropology and Identification of Human Remains" no CEMEL, financiado pelo Global Opportunities Fund do Governo Bri- tânico. Este projeto viabilizou a criação do Laboratório de Antropologia Forense no CEMEL e a elaboração de um protocolo para análise de ossadas, o que permitiu a realização de um trabalho de qualidade e confiabilidade na área médico-legal, com valor social intrínseco, além de sua relevância científica.

O protocolo elaborado inicialmente utilizava um software de edição de texto, como Microsoft Word®, cujo preenchimento era demorado, principalmente porque cálculos necessários ao exame antropológico eram realizados manualmente e incluídos como texto. Além disso, o cruzamento das informações dos diversos relatórios com dados de possíveis vítimas também era realizado manualmente, em um processo demorado que gerava atraso nos resultados.

Com o intuito de facilitar o registro, o armazenamento e a análise dos dados antropológicos coletados, cogitou-se a transformação deste protocolo para análises de ossadas em um software. Como outras vantagens possíveis, um software apresentaria ainda possibilidade de maior segurança, visto que o mesmo poderia ser criado para utilização apenas por usuários cadastrados e pela proteção através de senha individual. O correto funcionamento deste software viabilizaria também, em médio prazo, a sua distribuição para outros centros de atividades médico-legais, de forma a ampliar a utilização das técnicas de Antropologia Forense.

Assim, o objetivo almejado foi a criação de um software de baixo custo para facilitar o registro, a análise, a comparação e garantir a segurança de dados de exames antropológicos de ossadas no CEMEL da FMRP-USP.

\section{2- MATERIAIS E MÉTODOS}

A primeira etapa para a transformação do protocolo para análise de ossadas no software LAF 1.0 foi definir o formato que o mesmo seria elaborado e, a partir de então, escolher as ferramentas computacionais adequadas.

Para viabilizar a criação de um software Web, cuja finalidade é prover a formação de redes de computadores e aumentar a capacidade de cruzamento de informações, escolheu-se um servidor gratuito e de elevada confiabilidade, o Apache ${ }^{\circledR}{ }^{4}$.

Para a linguagem computacional foi utilizado o PHP® (Hypertext Preprocessor), devido à sua distribuição gratuita e à sua capacidade de processar todas as informações fornecidas pelo usuário ${ }^{5}$. 
O MySQL® foi o Sistema Gerenciador de Banco de Dados escolhido para armazenar todo o conteúdo processado. Esta escolha se justificou pela eficiência deste banco de dados, que tem baixa necessidade de memória computacional para o processamento dos dados e execução de cálculos, além de também ser gratuito 6 .

\section{3- RESULTADOS}

\section{1- Banco de Dados}

Com a finalidade de agilizar e facilitar o armazenamento e a consulta às informações, foi construído um banco de dados com uma arquitetura extremamente interligada, baseada em chaves estrangeiras e identificadores auto-incrementáveis para a conexão entre tabelas. Este diagrama mostrou-se satisfatório aos testes executados e não apresentou erros de armazenamento nem de recuperação de dados durante os testes realizados.

O Banco de Dados é formado pelas seguintes seções:

- Tabela Ossada: tabela principal onde o número do laudo iniciado é armazenado e apresenta também um contador auto-incrementável, para codificação e armazenamento seguro dos laudos, além da chave para tabela Funcionário (chave estrangeira).

- Tabela Funcionário: agrupa todos os funcionários, usuários ou não, cadastrados no software.

- Tabelas Crânio, Axial, Costela e Pelve e Apêndices: armazenam dados relativos ao inventário do esqueleto. Todas possuem a chave da tabela Ossada como chave estrangeira.

- Tabela Fotos: além de possuir a chave da tabela Ossada como chave estrangeira, armazena o endereço das fotos inseridas no disco rígido do servidor, assim como legendas, categoria da foto, número de fotos da mesma categoria por laudo, entre outros.

- Tabela Descrições Ossadas: armazena informações relativas a itens que descrevam a ossada encontrada, como causa da morte, compleição, cabelo, sexo, entre outros. Também possui como chave estrangeira a chave da tabela Ossada.

- Região Determinação do Sexo: agrupa duas tabelas relativas ao armazenamento de informações utilizadas na determinação do sexo da ossada.

- Região Determinação da Idade: também contém duas tabelas que são utilizadas para a estimativa de idade.
- Região Estatura e Destreza Manual: armazena dados relativos à determinação da estatura e da destreza manual.

\section{2- Segurança}

Como forma de segurança, alguns mecanismos foram desenvolvidos para impedir que os dados possam ser corrompidos ou acessados por indivíduos não autorizados.

O primeiro mecanismo é permitir que apenas IPs autorizados acessem o web site do programa. Cabe salientar que IP é o acrônimo da expressão inglesa Internet Protocol (Protocolo de Internet) que é um tipo de comunicação utilizada em rede para encaminhamento de dados entre dois computadores ${ }^{7}$. O segundo mecanismo é tornar cada usuário único, com uma senha pessoal, criptografada pelo banco de dados.

\section{3- Interface}

A interface gráfica foi escolhida para deixar um visual limpo e agradável ao usuário (Figura 1).

\section{4- Testes}

Testes com dados não reais apresentaram sucesso no cruzamento de dados com as informações no banco de dados do sistema, indicando posteriormente o perfeito acoplamento com dados reais.

\section{4 - CONCLUSÕES}

O software criado recebeu inicialmente a designação de LAF 1.0 (acrônimo para Laboratório de Antropologia Forense). Após realização de testes com dados reais e opiniões dos usuários, o mesmo recebeu algumas atualizações e melhorias e encontra-se, atualmente, na versão 2.0. Verificou-se ainda que, graças à metodologia usada na sua elaboração, este software permanece facilmente acessível para incrementos e melhorias no seu desempenho, assim como para o aperfeiçoamento de interface e reutilização de códigos com vista a melhores resultados por parte de seus autores.

A versão do software LAF 1.0 foi registrada no Instituto Nacional de Propriedade Intelectual (INPI) em quinze de fevereiro de dois mil e sete, sob o número 014854 e poderá ser distribuído gratuitamente aos Institutos Médico-Legais e outros centros afins que tiverem a intenção de utilizá-lo para implantação de um serviço de Antropologia Forense. 


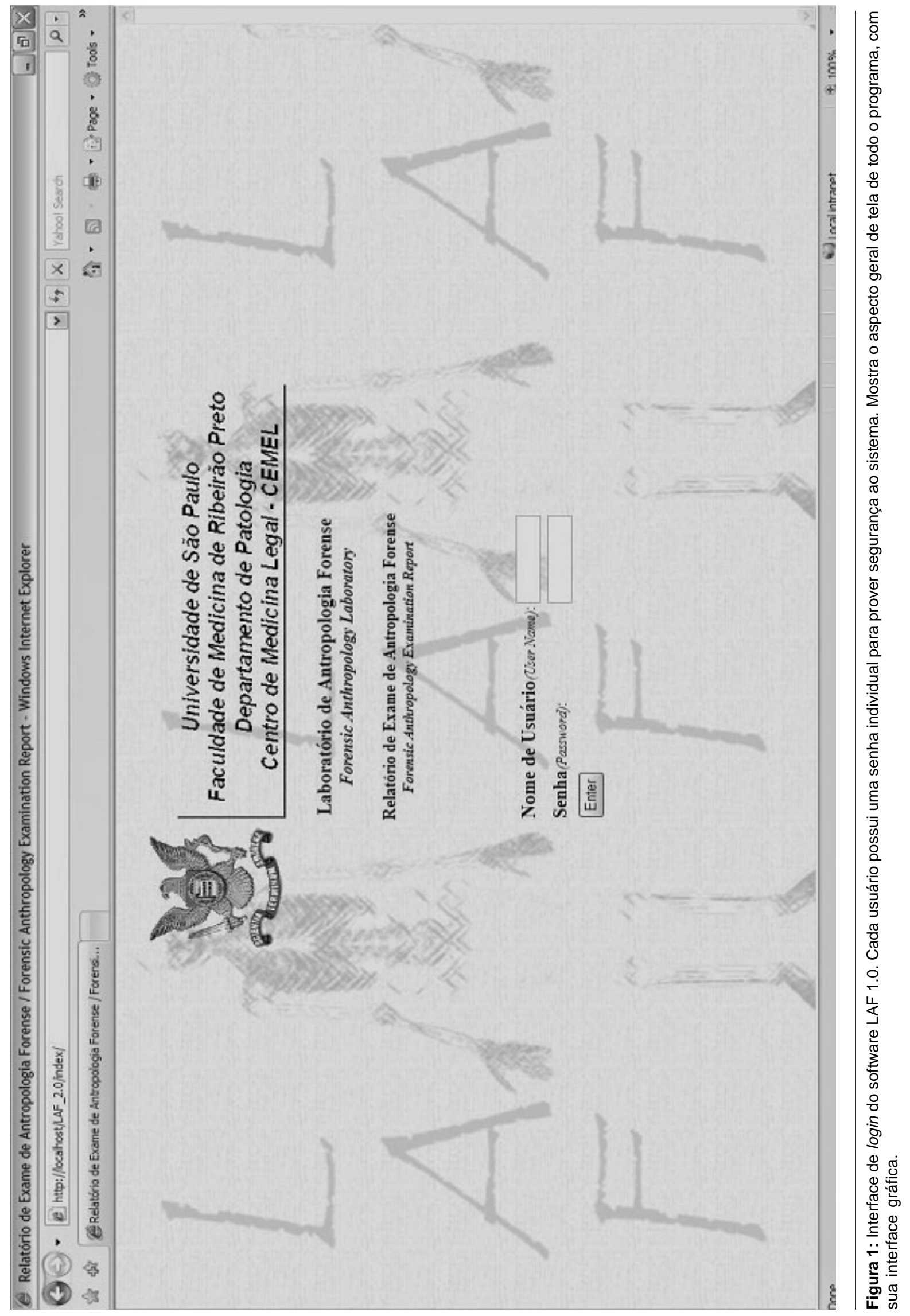


Crocco LG, Brondi GO, Soares ATC, Felipe JC, Evison MP, Guimarães MA. LAF 1.0: establishment of a computerized system for laboratories of Forensic Anthropology. Medicina (Ribeirão Preto) 2008; 41 (1): 12-16.

ABSTRACT: Objective: A Software development for registration and recovery of information on Forensic Anthropology, based on the protocol developed during the project "UK - Brazil Scientific Cooperation - Forensic Anthropology and Identification of Human Remains". Methods: Considering it is a Browser accessed application (software that allows Internet access, as Microsoft Internet Explorer $\left.{ }^{\circledR}\right)$, it was necessary to choose an adequate programming language to this requirement as the server application. The chosen language was $\mathrm{PHP} \AA$ and the server application was Apache®. For data storage it was chosen the Data Bank Managing System MySQL $\AA$. Results: Tests performed with real data proved the LAF 1.0 efficiency, considering safety, reliability in storing and recovering data, as well as user satisfaction with clean, pleasant interfaces. This version was registered at the National Institute of Intellectual Property (INPI) and it is available for use in all medico-legal services interested in the creation of a Forensic Anthropology laboratory. Conclusions: The software improoved the generation of anthropological examination reports at Medico Legal Centre (CEMEL) and allowed the creation of a computerized tool for use in similar services, which will permit easiness in personal capacitating in Forensic Anthropology. Nowadays the software is in its updated version designated as LAF 2.0.

Keywords: Forensic Medicine. Forensic Anthropology. Bones. Software.

\section{5- REFERÊNCIAS}

1 - Brasil. Ministério da Saúde. Vigilância Epidemiológica em Acidentes e Violências. Disponível em: http://portal.saude.gov.br/ portal/svs/visualizar_texto.cfm?idtxt=21853, acessado em 31 de julho de 2007.

2 - Martin CC, Melki JA, Guimarães MA. Assessment of methods of homicides in a Brazilian city: a preliminary study. Forensic Sci Int 1999; 22: 106(1):19-25.

3 - Adam D. Back from the dead. Nature 2003; 423: 13-14.

4 - The Apache Software Foundation. Disponível em http:// www.apache.org. Acessado em 27 de julho de 2007.
5 - PHP: Hypertext Preprocessor. Disponível em http:// www.php.net. Acessado em 27 de julho de 2007.

6 - MySQL AB: The world's most popular open source database. Disponível em http://www.mysql.com. Acessado em 28 de julho de 2007.

7 - IPsec. Disponível em: http://pt.wikipedia.org/wiki/IPSec. Acessado em 28 de julho de 2007.

Recebido para publicação em 27/08/2007

Aprovado para publicação em 13/05/2008 